\title{
Retail Channels Choice under Competition: Platform-Sell or Traditional Resell
}

\author{
Xiaojing $\mathrm{Li}^{1}$ and Jianhua $\mathrm{Ma}^{2}$ \\ ${ }^{1}$ School of Business, Sichuan Normal University, Chengdu 610101, China \\ ${ }^{2}$ Colleage of Managemet, Shen Zhen University, Shen Zhen 518060, China \\ jing811207@163.com,majianhuamail@126.com
}

\begin{abstract}
This paper investigates retailers' channels choice in a two-tier supply chain system consisting competing manufacturers and competing retailers. Each manufacturer produces a single substitutable product, and sells it through two common retailers. Retailers, as Stackelberg leaders, may offer two different channel structures to manufacturers, either traditional retailing or platform retailing. We compare four channel configurations and seek for a subgame Nash equilibrium. Our results show that, without product service, the equilibrium channel structure of the retailers would move from the platform retailing to traditional channel as the product competition intensifies and the platform retailing is a dominant equilibrium strategy. Conversely, while the product service is offered, the platform retailing is a unique Nash equilibrium, and it may push the retailers into the prisoner's dilemma.
\end{abstract}

Keywords: competing supply chain; Stackelberg game; cross sale, Nash equilibrium

\section{Introduction}

There has been growing interest in providing retail platform for manufacturers both in brick and mortar stores and on the internet. Traditional giant retailers, such as Wal-Mart, Macy and Bloomingdale, rent out retail space to manufacturers and give them complete autonomy over retailer decisions so that manufactures can sell their own brand products, typically cosmetics and apparel, directly to the consumers. On the internet, Taobao, a dominant online retailer in China, provides a platform for small businesses and individual entrepreneurs to open online stores which attract tens of thousands of active buyers. The retail platform, as a two side market, simultaneously serves manufacturers and consumers. Comparing to establishing a costly direct channel, manufactures could reach out to consumers and control the retail price with a lower cost while selling through the retail platform. And retailers can earn steady and sustainable profit from charging the platform fee instead of facing the market risks of reselling.

Even though the retail platform is a huge trend, there are still some manufacturers, which have lower brand awareness and is vulnerable to the market risks, preferring traditional retailing, where manufactures only need to wholesale the products to retailers. In order to expand the variety and range of the products, it is common for retailers, such as Amazon and JD.com, to provide a retail platform for the third part sellers while reselling products of certain categories themselves simultaneously. And according to the data in Table 1, among different categories, the percentage of products sold by JD.com itself varies greatly, for instance, JD.com resells $70 \%$ of the whole products in its "Electronics" category while reselling only 5\% in its "Toys \& Games" category. The details of total five categories are shown below(see Table 1).

Received (April 1, 2017), Review Result (October 11, 2017), Accepted (January 12, 2018) 
Table 1. Percentage of Products sold by JD.com in Some Sample Product Categories

\begin{tabular}{cccc}
\hline Category & $\begin{array}{c}\text { Total\# of } \\
\text { products }\end{array}$ & $\begin{array}{c}\text { \%sold by } \\
\text { JD.com }\end{array}$ & \%sold by third-party seller \\
\hline Electronics & 1024550 & 70 & 30 \\
\hline Cosmetics & 753410 & 17 & 83 \\
\hline Toys \& Games & 15760 & 5 & 95 \\
Sports \& & 40714 & 8 & 92 \\
Outdoors & & & 97 \\
Shoes & 57823 & 3 & \\
\hline
\end{tabular}

For both brick and mortar stores and online retailers, there are two distribution channels which retailers can offer to manufactures: one is traditional retailing, where retailers order products from manufactures and resell to customers; the other is retail platform, where retailers authorize manufactures to sell through the retail platform and claims a platform fee. The discussion above naturally leads to several questions: What factors play the main role of retailers' choosing distribution channels (traditional retailing or retail platform) and is there any difference between the supply chain members' decision making under different distribution channels?

And it is also necessary to discuss the retailers' distribution channel choice while both retailers and manufacturers facing horizontal competition since it is more in line with current market status. Taken e-book market for example, Amazon was the first to resell ebooks on Kindle. Apple also starts reselling e-books in iBook store correspondingly, and furthermore, Apple upgrade the iBook store to an e-book retail platform so that independent publishers can sell their own books. The competition between Apple and Amazon severely sways the cooperation and reselling agreement between Amazon and the publishers and finally a compromise of establishing a same retail platform is reached after a round of negotiations. (Stone and Rich, 2010). The example above told us that retailer's distribution channel choice is affected by both the competitor's distribution channel choice and the intensity of competition. Some new questions arise: while both the manufacturers and the retailers face horizontal competition in the market, which distribution channel should the retailers choose? How does the channel choice evolve over time and what factors drive this evolution?

To develop an in-depth understanding of these above-mentioned questions, we establish a supply chain model with two competing manufacturers and two competing retailers. Each manufacturer produces a substitutable product (different brands but in the same category) and sells it through two common retailers. The market features manufacturer competition, represented by product substitution; and retail competition, represented by store substitution. Similar competitive structure is commonly observed in practice. For instance, competing fashion manufacturers, such as Calvin Klein and Ralph, both sell their products through Macy and Lord \&Taylor, which is two of the largest department stores. $P \& G$ and Unilever produce substitutable personal care products and sell through duopoly common retailers, such as Amzon and JD.com. As the Stacklberg leader, retailers have two distribution channels to choose. One is the traditional retailing where retailers order products and resell to consumers; the other is the retail platform where manufacturers sell products directly on retailers' platform and pay a platform fee to retailers. The main contributions of our paper are as follows.

(1) So far, the cross sale is still a key problem in supply chain applications. What's more, there are no published works that investigate how to design the channel structure in competing supply chain with cross sale. We develop competing supply chain models to analyze equilibrium strategy of channel structure choice game with cross sale in this paper. 
(2) We consider that the market features both manufacturer competition, represented by product substitution, and retail competition, represented by store substitution. Similarly, competitive structure is commonly observed in practice. Our objective is to provide management insights into channel structure choice and cross sale on the competing supply chain.

(3) We refer to three different types of contracting arrangements: TT, TP and PP, where $\mathrm{T}$ denotes traditional channel and $\mathrm{P}$ denotes platform retailing. We establish the Stackelberg game models and derive members' decision behavior under these three configurations without considering the product service and gain some interesting management insights.

(4) We not only build the competitive supply chain model to analyze the impact of horizontal competition on channel choice, but also extend this model to discuss the best channel structures for retailers while considering the produce service. We find that the platform retailing will be the only option for retailers with the product service offered, but sometimes the platform retailing is not the best option for retailers.

(5) We design the platform retailing mechanism by adopting the different perspectives and derive the coordination conditions with platform retailing in competing supply chain with cross sale.

The remainder of this paper is organized as follows. In the next section, we briefly introduce current literature and describe the model setup in Section 3. The formulations and solutions of four possible channel combinations: TT, TP, PT, and PP are present in Section 4. In Section 5, we discuss the platform fee mechanism, and identify retailers' equilibrium channel choice and the evolution of the channel choice under different conditions. In Section 6, we establish the channel choice model while considering product service. In Section 7, we provide a numerical simulation to illustrate the impact of horizontal competition and product service on the decision variables. Finally, we summarize the conclusions and suggest possible directions for future research in Section 8.

\section{Literature}

Our work is related to two previous streams of research. The first develops the implications of direct channel for supply chain participants and the second studies various aspects associated with competing supply chain. We discuss each of these streams in order.

Prior research on direct channel has paid a lot of attention to the impact of introducing the direct channel to the traditional channel structure. Balasubramanian (1998) shows that direct channel's entry leads to the competition transitions form two traditional retailers to a traditional retailer and a direct seller. Lal(1999), Brynjolfsson(2000), Zettelmeyer(2000) and Cattani et al., (2006) discuss the price strategy of dual-channel supply chain. Chiang et al., (2003) suggest that establishing a direct channel enhances traditional manufacture's bargaining power in the vertical supply chain. Yoo et al. (2011) and Ofek et al. (2011) obtain that direct channel entry for a retailer does not always lead to lower retail prices and higher consumer welfare, and it might generate smaller profit under certain conditions. There are several papers considering the retail platform as a distribution channel. Jiang et al., (2011) suggest there are two types of independent sellers on platform - one with high demand and the other with low demand. It uses a game-theoretic model to investigate the participants' strategic behavior of the one (manufacturer)-to-one (retailers) supply chain. Abhishek et al., (2013) consider a manufacture sells products through one traditional retailer and two symmetric electronic retailers. It recognizes the effect of e-tailer's channel choice (traditional reselling or the platform retailing) on supply chain members. However, the papers above mentioned only focus on the supply chain structure with one (manufacturer)-to-one (retailer) or one (manufacturer)-to-many 
(retailers). Different form them, our paper investigates two (manufacturers)-to-two (retailers) structure, which means both competitive manufacturers and competitive retailers in the market. Moreover, we consider the competitive manufacturers sell their products through two common retailers, which is cross sale.

The other related stream of research is on chain-to-chain or channel-to-channel competition. McGuire and Staelin(1983) investigate the effect of product substitutability on Nash equilibrium distribution structures in a duopoly where each manufacture distributes its goods through a single exclusive retailer. Coughlan(1985), Moorthy (1988,2005), Bernstein and de Ve' ricourt(2008), and $\mathrm{Ha}(2008), \mathrm{Ai}(2012)$ extend the McGuire model form different perspectives, however, these literatures only focus on exclusively competitive supply chain structure. The core of our paper arises from the aforementioned market structure is that competing manufacturers sell products to different retailers instead of the exclusive one, and competing retailers also order products from different manufacturers simultaneously. Trivedi (1998) and Cai(2012) considers the selling format of cross sale, but there are several limitations in their paper, for example, it only considers the product substitutability and does not consider the product service offered by supply chain members and the effect of service level on channel choice. Differently from them, our research focuses on the choice of distribution channel (retail platform and traditional channel) under cross sale supply chain. We discuss the design of service level and the platform fee under retail platform format and develop the effect of horizontal competition and product service on distribution channel choice.

\section{Assumptions}

We consider a two-tier supply chain system consisting of two competing manufacturers (e.g., $\mathrm{P} \& \mathrm{G}$ and Unilever), denoted by $\mathrm{M} 1$ and $\mathrm{M} 2$, and two competing retailers (e.g,, Amzon and JD.com), denoted by R1 and R2. Each manufacture produces a single product (i.e., manufacture $i$ produces personal care product $i, i=1,2$ ), and each retailer provides a single channel (i.e., retailer $x$ provides channel $x, x=1,2$ ). Therefore, there are $2 \times 2=4$ possible channel configurations, which is detailed below.

1. TT: Both retailers order products from both manufactures and resell to consumers.(for example, both $\mathrm{P} \& \mathrm{G}$ and Unilever wholesale their personal care products to two retailers and retailers resell them to consumers )

2. TP: retailer1 orders products from both manufactures for reselling, and retailer 2 offers a platform to both manufactures. (for example, $\mathrm{P} \& \mathrm{G}$ and Unilever establish their store on Amazon to sell their own personal care products; on the other hand, at the same time, $P \& G$ and Unilever wholesale their personal care products to JD.com.)

3. PT: retailer 1 offers a platform to both manufactures, and retailer 2 orders products from both manufactures for reselling. (for example, $P \& G$ and Unilever establish their store on JD.com to sell their own personal care products; on the other hand, at the same time, $P \& G$ and Unilever wholesale their personal care products to Amazon.)

4. PP: Both retailers offer a platform to both manufactures. (for example, $P \& G$ and Unilever establish their store both on JD.com and Amazon to sell their own personal care products)

Note that the Configuration TP and PT are symmetric, so we just consider one of them, the Configuration TP hereafter. Similarly, due to the symmetry, the equilibrium solution of manufacturer $i$ is same as manufacturer $j$, so we just discuss manufacturer $i$ 's problem.

Let $p$ represent the retail price and $q$ the demand. Consistent with the existing literature (e.g., Trivedi 1998, Feng2012), we assume that the demand for manufacturer $i$ 's product sold by retailer $x$ is given by

$$
\begin{aligned}
& p_{i x}=a-\left(q_{i x}+\beta q_{i y}\right)-\gamma\left(q_{j x}+\beta q_{j y}\right) \\
& \text { such that } i=1,2 ; x=1,2 ; j=3-i ; y=3-x .
\end{aligned}
$$


Where $0<\gamma<1$ represents the degree of product substitutability within a retailer, and $0<\beta<1$ represents the degree of store substitutability for the same product. In addition, in the rest of the paper, $\pi_{n}^{h}$ denotes the profit function for supply chain member $n$ in configuration $h$, Superscript $h$ takes the values of TT, TP, PT, PP, respectively. Subscript $n$ takes the values of $M i, M j, R i$ and $R j$, denoting the manufacturer $i$, manufacturer $j$, retailer $x$, and retailer $y$, respectively. $p_{u v}^{h}$ and $w_{u v}^{h}$ denote the retail price and wholesale price of the product which manufacturer $u$ sells to retailer $v$ in configuration $h$. Subscript $u$ takes the values of $i, j$,denoting the manufacturer $i$ and $j$. Subscript $v$ takes the values of $x, y$, denoting the manufacturer $x$ and $y$.

The sequence of decisions is as follows: first, the distribution channel (traditional retailing or retail platform) is offered by the retailers to two manufacturers. If the traditional channel is offered by the retailer, the manufacturer will give a wholesale of products to achieve maximum profit. If the retail platform is offered, the platform fee is charged by retailer. And then, the manufacturer decides retail price. Note that all supply chain members have access to the same information while maximizing their own profit in a Nash game, and the game setting is referred to as independent ownership competition. (Economides and Salop 1992, Ingene and Parry 2004).

\section{Competing Supply Chain Model without Product Service}

In this section, we present the general formulations and solutions to competitive supply chain model under three configurations of the channel structure-PP, TP and TT. Insights are developed in terms of pricing behaviors of supply chain members, and strategic interaction under different competitive intensity.

\subsection{Both Retailers Offer Traditional Distribution Channel (TT)}

In this configuration, two retailers both purchase products with wholesale price from both manufacturers. The manufacturers decide the wholesale price of products firstly. Then, the retailers choose the retailer price by taking account of the wholesale price. Manufacturer $i$ 's and retailer $x$ 's profit functions can be written as

$$
\begin{aligned}
& \pi_{M i}=w_{i x} q_{i x}+w_{i y} q_{i y} . \\
& \pi_{R x}=\left(a-q_{i x}-\beta q_{i y}-\gamma q_{j x}-\gamma \beta q_{j y}-w_{i x}\right) q_{i x}+\left(a-q_{j x}-\beta q_{j y}-\gamma q_{i x}-\gamma \beta q_{i y}-w_{j x}\right) q_{j x} .
\end{aligned}
$$

Such that $i=1,2 ; j=3-i ; x=1,2 ; y=3-x$.

From equation (3), we can derive that

$$
\begin{aligned}
& \frac{\partial \pi_{R x}\left(q_{i x}, q_{j x}\right)}{\partial q_{i x}}=a-2 q_{i x}-w_{i x}-2 q_{i y} \beta-q_{j x} \gamma-q_{j y} \beta \gamma \\
& \frac{\partial^{2} \pi_{R x}\left(q_{i x}, q_{j x}\right)}{\partial q_{i x}{ }^{2}}=-2 \quad, \quad \frac{\partial \pi_{R x}\left(q_{i x}, q_{j x}\right)}{\partial q_{j x}}=a-2 q_{j x}-w_{j x}-2 q_{j y} \beta-q_{i x} \gamma-q_{i y} \beta \gamma \\
& \frac{\partial^{2} \pi_{R x}\left(q_{i x}, q_{j x}\right)}{\partial q_{j x}{ }^{2}}=-2, \frac{\partial^{2} \pi_{R x}\left(q_{i x}, q_{j x}\right)}{\partial q_{i x} \partial q_{j x}}=-\gamma \frac{\partial^{2} \pi_{R x}\left(q_{i x}, q_{j x}\right)}{\partial q_{j x} \partial q_{i x}}=-\gamma .
\end{aligned}
$$

Thus, the Hessian Matrix of the retailers' profit function in model TT without considering service is given by 


$$
\left[\begin{array}{ll}
\frac{\partial^{2} \pi_{R x}\left(q_{i x}, q_{j x}\right)}{\partial q_{i x}{ }^{2}} & \frac{\partial^{2} \pi_{R x}\left(q_{i x}, q_{j x}\right)}{\partial q_{i x} \partial q_{j x}} \\
\frac{\partial^{2} \pi_{R x}\left(q_{i x}, q_{j x}\right)}{\partial q_{j x} \partial q_{i x}} & \frac{\partial^{2} \pi_{R x}\left(q_{i x}, q_{j x}\right)}{\partial q_{j x}{ }^{2}}
\end{array}\right]=\left[\begin{array}{ll}
-2 & -\gamma \\
-\gamma & -2
\end{array}\right] .
$$

Obviously, this matrix is said to be negative definite. It follows that $\partial \pi_{R x}\left(q_{i x}, q_{j x}\right)$ is a strictly concave function of $q_{i x}$ and $q_{j x}$. Let $\partial \pi_{R x}\left(q_{i x}, q_{j x}\right) / \partial q_{i x}=0$, we can deduce the equation as:

$$
\widehat{q}_{i x}=\left(a(1-\gamma)-\beta\left(1-\gamma^{2}\right) q_{i y}-w_{i x}+\gamma w_{j x}\right) /\left(2\left(1-\gamma^{2}\right)\right)
$$

Using the same way, we can derive reaction function $\hat{q}_{i y}$. The manufacturers use reaction functions $\widehat{q}_{i x}, \widehat{q}_{i y}$ in profit function (Equation (2)) to determine optimal margins using first-order conditions, thus getting,

$$
\widehat{w}_{i x}=a / 2+c / 2-\left(q_{i y} \beta\right) / 2+\left(w_{j x} \gamma\right) / 2-(a \gamma) / 2+\left(q_{i y} \beta \gamma^{2}\right) / 2
$$

Solving the above equation for $\widehat{q}_{i x}, \widehat{w}_{i x}$, and then substituting into demand and profit function, we get equilibrium price and profits, shown in Table 1.

\subsection{Both Retailers Offer the Platform To Manufacturers (PP)}

In this configuration, both retailers propose the retail platform and both manufacturers accept it. We follow a decision rule like this. First, each retailer charges the platform fee. Second, both manufacturers accept the platform fee and decide the retail price simultaneously. Then, retailer $x$ and $y$ 's profits are $2 F$. Manufacturer $i$ 's profit function is

$$
\pi_{M i}=\left(a-q_{i x}-\beta q_{i y}-\gamma q_{j x}-\gamma \beta q_{j y}\right) q_{i x}+\left(a-q_{i y}-\beta q_{i x}-\gamma q_{j y}-\gamma \beta q_{j x}\right) q_{i y}-2 F \text { (6) }
$$

From equation (6), we can derive that

$$
\begin{aligned}
& \frac{\partial \pi_{M i}\left(q_{i x}, q_{i y}\right)}{\partial q_{i x}}=a-2 q_{i x}-2 q_{i y} \beta-q_{j x} \gamma-q_{j y} \beta \gamma, \frac{\partial^{2} \pi_{M i}\left(q_{i x}, q_{i y}\right)}{\partial q_{i x}{ }^{2}}=-2, \\
& \frac{\partial \pi_{M i}\left(q_{i x}, q_{i y}\right)}{\partial q_{i y}}=a-2 q_{i y}-2 q_{i x} \beta-q_{j y} \gamma-q_{j x} \beta \gamma, \frac{\partial^{2} \pi_{M i}\left(q_{i x}, q_{i y}\right)}{\partial q_{i y}{ }^{2}}=-2, \\
& \frac{\partial^{2} \pi_{M i}\left(q_{i x}, q_{i y}\right)}{\partial q_{i x} \partial q_{i y}}=-\gamma, \frac{\partial^{2} \pi_{M i}\left(q_{i x}, q_{i y}\right)}{\partial q_{i y} \partial q_{i x}}=-\gamma .
\end{aligned}
$$

Thus, the Hessian Matrix of the retailers' profit function in model PP without considering service is given by

$$
\left[\begin{array}{ll}
\frac{\partial^{2} \pi_{M i}\left(q_{i x}, q_{i y}\right)}{\partial q_{i x}{ }^{2}} & \frac{\partial^{2} \pi_{M i}\left(q_{i x}, q_{i y}\right)}{\partial q_{i x} \partial q_{i y}} \\
\frac{\partial^{2} \pi_{M i}\left(q_{i x}, q_{i y}\right)}{\partial q_{i y} \partial q_{i x}} & \frac{\partial^{2} \pi_{M i}\left(q_{i x}, q_{i y}\right)}{\partial q_{i y}{ }^{2}}
\end{array}\right]=\left[\begin{array}{ll}
-2 & -\gamma \\
-\gamma & -2
\end{array}\right] .
$$

Obviously, this matrix is said to be negative definite. It follows that $\partial \pi_{M i}\left(q_{i x}, q_{i y}\right)$ is a strictly concave function of $q_{i x}$ and $q_{i y}$. Let $\partial \pi_{M i}\left(q_{i x}, q_{i y}\right) / \partial q_{i x}=0$, we can deduce the equation as:

$$
\tilde{q}_{i x}=\left(a-c-\beta(1+\gamma) q_{i y}\right) /(2(1+\gamma))
$$


Solving the above equation for $\tilde{q}_{i x}$, and then substituting into demand and profit function, we get equilibrium price and profits, shown in Table 1.

\subsection{Retailer1 Offer A Platform and Retailer 2 offer a Traditional Channel (TP)}

In this channel arrangement, one of the retailers (i.e., $x$ ) acts as a reseller and the other retailer $y$ offers retail platform. We follow a decision rule like this. In the first stage, retailer $y$ charges the platform fee to both manufacturers. Then, both manufacturers decide the wholesale price of traditional channel and the retail price of platform selling to maximize its total profits. At last, retailer $x$ decides the reselling price. In the second stage, for given outcome resulting from stage one. The manufacturer and retailer profit functions are

$$
\begin{aligned}
& \pi_{M i}=w_{i x} q_{i x}+\left(a-q_{i y}-\beta q_{i x}-\gamma q_{j y}-\gamma \beta q_{j x}\right) q_{i y}-F \\
& \pi_{R x}=\left(a-q_{i x}-\beta q_{i y}-\gamma q_{j x}-\gamma \beta q_{j y}-w_{i x}\right) q_{i x}+\left(a-q_{j x}-\beta q_{j y}-\gamma q_{i x}-\gamma \beta q_{i y}-w_{j x}\right) q_{j x} .
\end{aligned}
$$

Because equation (9) is the same as equation (3), we uses the reaction function (4) in the profit function (Equation (8)), to determine optimal retail price by first-order conditions, thus getting,

$$
\hat{w}_{i x}=a(1-\gamma) / 2+c / 2+\gamma w_{i y} / 2-\beta q_{i y} / 2-\gamma^{2} \beta q_{i y} / 2
$$

Using the same way as model TT and PP, we can derive reaction functions $\hat{w}_{i y}, \hat{p}_{j x}, \hat{p}_{j y}$. Solving above equations, we can obtain equilibrium values of prices and profits (see Table 2).

Table 2. The Equilibrium Prices and Profits without Product Service

\begin{tabular}{|l|l|l|l|}
\hline & TT & TP & PP \\
\hline$w_{i x}$ & $\frac{2 a}{\beta-2 \gamma+4}$ & $\frac{a\left(4-2 \beta-2 \gamma-2 \gamma^{2}+2 \beta \gamma^{2}\right)}{(\gamma-2)\left(\beta^{2} \gamma-2 \gamma+\beta^{2}-4\right)}$ & \\
\hline$w_{i y}$ & $\frac{2 a}{\beta-2 \gamma+4}$ & & \\
\hline$q_{i x}$ & $\frac{a}{\beta+2 \gamma+\beta \gamma-2 \gamma^{2}+4}$ & $\frac{a(\gamma-\beta-\beta \gamma+2)}{(\gamma+1)(\gamma-2)\left(\beta^{2} \gamma-2 \gamma+\beta^{2}-4\right)}$ & $\frac{a}{2 \beta+\gamma+\beta \gamma+2}$ \\
\hline$q_{i y}$ & $\frac{a}{\beta+2 \gamma+\beta \gamma-2 \gamma^{2}+4}$ & $\frac{a(2-\beta)}{2 \gamma-\beta^{2} \gamma-\beta^{2}+4}$ & $\frac{a}{2 \beta+\gamma+\beta \gamma+2}$ \\
\hline$\pi_{M i}$ & $\frac{4 a^{2}(1-\gamma)}{(\gamma+1)(\beta-2 \gamma+4)^{2}}$ & $\frac{\left((\beta-2)(\gamma-2)(\gamma+1)\left(\beta^{2}\left(\gamma^{2}-1\right)-\beta \gamma^{2}-2 \gamma+4\right)+2(1-\gamma)(\beta-\gamma+\beta \gamma-2)^{2}\right) a^{2}}{(\gamma+1)(\gamma-2)^{2}\left(2 \gamma-\beta^{2} \gamma-\beta^{2}+4\right)^{2}}-F$ & $\frac{2 a^{2}}{(\gamma+2)^{2}(\beta+1)}-2 F$ \\
\hline$\pi_{M j}$ & $\frac{4 a^{2}(1-\gamma)}{(\gamma+1)(\beta-2 \gamma+4)^{2}}$ & $\frac{\left((\beta-2)(\gamma-2)(\gamma+1)\left(\beta^{2}\left(\gamma^{2}-1\right)-\beta \gamma^{2}-2 \gamma+4\right)+2(1-\gamma)(\beta-\gamma+\beta \gamma-2)^{2}\right) a^{2}}{(\gamma+1)(\gamma-2)^{2}\left(2 \gamma-\beta^{2} \gamma-\beta^{2}+4\right)^{2}}-F$ & $\frac{2 a^{2}}{(\gamma+2)^{2}(\beta+1)}-2 F$ \\
\hline$\pi_{R x}$ & $\frac{2 a^{2}}{(\gamma+1)(\beta-2 \gamma+4)^{2}}$ & $\frac{(\beta-\gamma+\beta \gamma-2)^{2} a^{2}}{(\gamma+1)(\gamma-2)^{2}\left(2 \gamma-\beta^{2} \gamma-\beta^{2}+4\right)^{2}}$ & $2 F$ \\
\hline$\pi_{R y}$ & $\frac{2 a^{2}}{(\gamma+1)(\beta-2 \gamma+4)^{2}}$ & $2 F$ & $2 F$ \\
\hline
\end{tabular}

Comparing the wholesale prices and sale quantities in different configurations, we can derive the proposition as follows.

\section{Proposition 1}

(i) $w_{i x}^{T T}>w_{i x}^{T P}\left(w_{i y}^{P T}\right)$

(ii) $q_{i x}^{T T}>q_{i x}^{T P}\left(q_{i y}^{P T}\right), q_{i y}^{T P}\left(q_{i x}^{P T}\right)>q_{i x}^{P P}, q_{i x}^{P P}>q_{i x}^{T T}$.

Proposition 1 demonstrates the sale quantity for the case that the both two retailers trade with manufacturer 1 and 2 by platform agreement is higher than that of the case that 
two retailers trade with manufacturer 1 and 2 by traditional channel. In addition, given that one retailer using a traditional channel, if the other shifts from a traditional channel to a retail platform, it will get a higher sale quantity than before. And the sequence of sale quantity under different scenarios is not influenced by product and store substitutability. What's more, it is also shown that no matter how intensive the horizontal competition is, the more retailers choose retail platform, the lower the wholesale price.

\section{Equilibrium Strategy of Channel Choice Game without Product Service}

In this section, we first present the solutions to determine the platform fee, and derive the impact of horizontal competition on platform fee. Then, we compare four configurations TT, TP, PT, and PP from retailer's point of view, and investigate which configuration can be observed in equilibrium.

\subsection{Design of the Platform Fee}

We first discuss the design of platform fee. In the above-mentioned retail platform, the manufacturer is required to pay a fixed fee to sell products on platform. To investigate the pure impact of channel choice on the members, we assume that when there is more than one supply chain to offer the platform fee, the fees offered by each chain are the same. Some prior literatures have the similar assumption, for example, Cai et al., (2012), Dukes et al., (2006), O’Brien and Shaffer $(1992,2005)$, and Horn and Wolinsky (1988).

With the case that retailer $x$ is a traditional retailer and retailer $y$ offers retail platform, if manufacturers accept the platform fee $F$ charged by retailer $y$, the destination configuration is TP; otherwise, they end up with configuration TT. When two retailers simultaneously offer the platform fee to manufacturer 1 and 2, then there are three possible results. The first is that two manufacturers both refuse retailer1 and 2's platform fee, thus they end up with configuration TT; the second is that one of retailer's platform fee $F$ is accepted and the other retailer's is refused, then the destination configuration of the negotiation is TP (PT); The third is that both manufacturers accept retailer1 and 2's platform fee $F$, configuration PP is formed.

In order to encourage manufacturers to accept the retail platform, the retailers always design the platform fee to make the manufacturer's profit at least same as the profit under traditional channel. In our paper, we assume if the retailers offer a retail platform, they can gain all the benefit of retail platform through the design of platform fee, since they are the leader of the market. In the other word, manufacturers' profits under the retail platform are same as those under traditional channels, which yields the following proposition

\section{Proposition 2}

$$
\begin{aligned}
& \text { (i) }\left\{\begin{array}{l}
\text { if } F=F_{a}, \text { then } \pi_{R y}^{T T}=\pi_{R y}^{T P} . \\
\text { if } F=F_{b}, \text { then } \pi_{R x}^{T P}=\pi_{R x}^{P P} . \\
\text { if } F=F_{c}, \text { then } \pi_{R x}^{T T}=\pi_{R x}^{P P} .
\end{array}\right. \\
& \text { Where }_{F_{a}=\frac{\left((\beta-2)(\gamma-2)(\gamma+1)\left(\beta^{2}\left(\gamma^{2}-1\right)-\beta \gamma^{2}-2 \gamma+4\right)+2(1-\gamma)(\beta-\gamma+\beta \gamma-2)^{2}\right) a^{2}}{(\gamma+1)(\gamma-2)^{2}\left(2 \gamma-\beta^{2} \gamma-\beta^{2}+4\right)^{2}}-\frac{4 a^{2}(1-\gamma)}{(\gamma+1)(\beta-2 \gamma+4)^{2}},}^{(\gamma+1)(\gamma-2)^{2}\left(2 \gamma-\beta^{2} \gamma-\beta^{2}+4\right)^{2}} \\
& F_{b}=\frac{2 a^{2}}{(\gamma+2)^{2}(\beta+1)}-\frac{\left((\beta-2)(\gamma-2)(\gamma+1)\left(\beta^{2}\left(\gamma^{2}-1\right)-\beta \gamma^{2}-2 \gamma+4\right)+2(1-\gamma)(\beta-2)^{2}\right) a^{2}}{\left(\gamma+a^{2}(1-\gamma)\right.}, \\
& F_{c}=\frac{a^{2}}{(\gamma+2)^{2}(\beta+1)}-\frac{2 \gamma+1)(\beta-2 \gamma+4)^{2}}{(\gamma+3}
\end{aligned}
$$


Proposition2 shows that the platform fees $F_{a}, F_{b}, F_{c}$ all depend on store substitutability $(\beta)$ and product substitutability $(\gamma)$. When one retailer (such as $x$ ) offer a retail platform and the other retailer $(y)$ moves form a traditional channel to a retail platform, the platform fee of retailer $x$ decrease, which is beneficial to the manufacturers. It implies as the number of retailers which offer retail platform increases, the platform fee charged by the retailer decreases, so the profit of the retail platform decreases. To some extent, it explains why so many retail platforms are free under severe competition and the oligopolistic retail platform could charge a high platform fee.
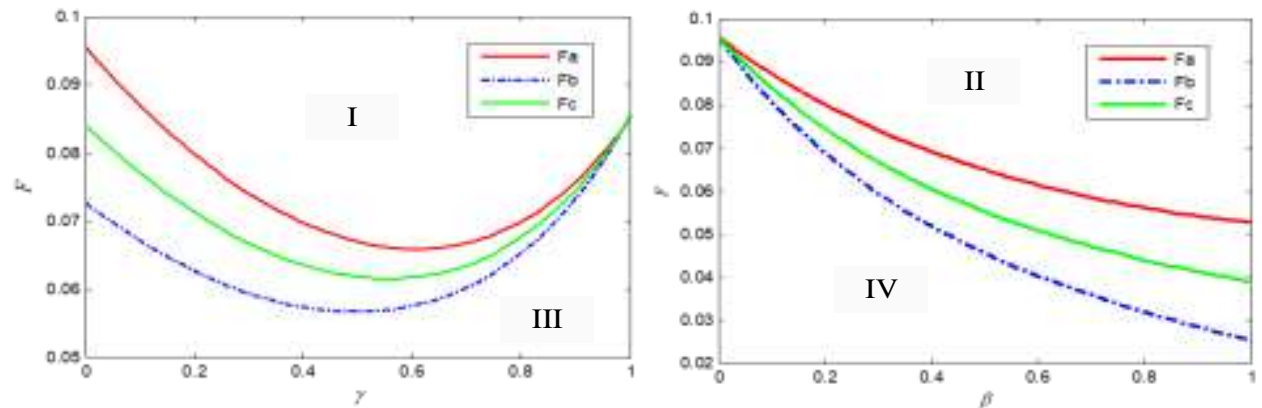

Figure 1. The Relationship of $F a, F b, F c$ and $\gamma, \beta$

In order to present above results visually, we use a numerical study. Setting $a=2, c=1, \gamma=0.3$ and $a=2, c=1, \beta=0.3$, the relationship of $F_{a}, F_{b}, F_{c}$ are shown in Fig1. Figure 1 suggests that in the shaded region I or II (the range $F>F_{a}$ ), the retailers in competing supply chain would prefer the retail platform. As $\gamma$ increases, this range increases first and then shrinks. As $\beta$ decreases, this range decreases gradually. Conversely, in the shaded III or IV (the range $F<F_{b}$ ), the retailers in competing supply chain would prefer the traditional channel, and the effect of $\gamma$ and $\beta$ on the range reverses.

\subsection{Equilibrium Channel Choice}

Now we turn our attention to the channel choice of retailers and explore in depth the effect of $\beta, \gamma$ on the equilibrium channel. In order to simplify the comparison, we propose several functions as,

$$
f_{a}(\gamma, \beta)=R_{y}^{T P}-R_{y}^{T T}, f_{b}(\gamma, \beta)=R_{x}^{P P}-R_{x}^{T P}, f_{c}(\gamma, \beta)=R_{x}^{P P}-R_{x}^{T T} .
$$

It is obvious that the rectangular area $\{(\gamma, \beta) \mid 0<\gamma<1,0<\beta<1\}$ is divided into four distinct regions L1, L2, L3, L4 by four curves: $f_{a}(\gamma, \beta)=0, f_{b}(\gamma, \beta)=0$, $f_{c}(\gamma, \beta)=0$.(see figure2). We can obtain:

$$
\begin{gathered}
L_{1}=\left\{(\gamma, \beta) \mid f_{a}(\gamma, \beta)>0, f_{b}(\gamma, \beta)>0, f_{c}(\gamma, \beta)>0, \text { and } 0<\gamma<1,0<\beta<1\right\} . \\
L_{2}=\left\{(\gamma, \beta) \mid f_{a}(\gamma, \beta)>0, f_{b}(\gamma, \beta)>0, f_{c}(\gamma, \beta)<0, \text { and } 0<\gamma<1,0<\beta<1\right\} . \\
L_{3}=\left\{(\gamma, \beta) \mid f_{a}(\gamma, \beta)<0, f_{b}(\gamma, \beta)>0, f_{c}(\gamma, \beta)<0, \text { and } 0<\gamma<1,0<\beta<1\right\} . \\
L_{4}=\left\{(\gamma, \beta) \mid f_{a}(\gamma, \beta)<0, f_{b}(\gamma, \beta)<0, f_{c}(\gamma, \beta)<0, \text { and } 0<\gamma<1,0<\beta<1\right\} .
\end{gathered}
$$

The following propositions evaluate the performance of traditional channel and retail platform in terms of the retailer's profit when the rival chooses different channel structures. 
Proposition 3 Given that one retailer $x$ offers a traditional channel, if $(\beta, \gamma) \in\left(L_{1} \cup L_{2}\right)$, then the other retailer $y$ has an incentive to shift form a traditional channel to a retail platform; if $(\beta, \gamma) \in\left(L_{3} \cup L_{4}\right)$, a traditional channel benefits retailer $y$.

Proposition3 indicates that when retailer $x$ chooses a traditional channel, if (1) the product substitutability $\gamma$ is not relatively high or (2) both the product substitutability $\gamma$ and the store substitutability $\beta$ are sufficiently high, retailer $y$ 's can have more profit under retail platform than traditional channel due to removing double marginalization. On the contrary, if the product substitutability $\gamma$ is relatively high and the store substitutability $\beta$ is low, the traditional channel is valuable to retailer $y$.

Proposition 4 Given that one retailer $x$ offers a retail platform, if $(\beta, \gamma) \in\left(L_{1} \cup L_{2} \cup L_{3}\right)$, the other retailer $y$ has an incentive to shift form a traditional channel to a retail platform; if $(\beta, \gamma) \in L_{4}$, a traditional channel benefits retailer $y$.

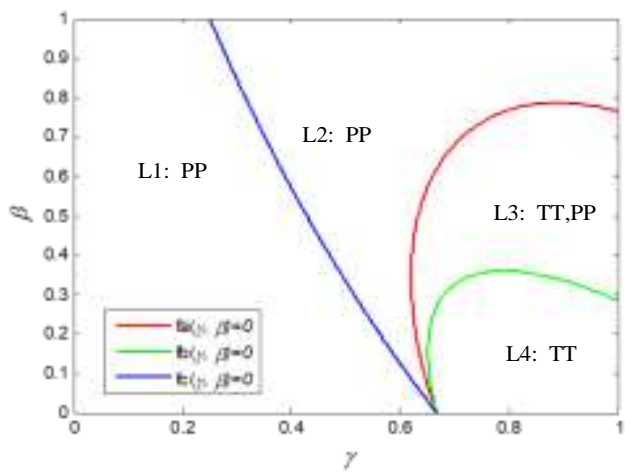

Figure 2. Equilibrium Channel Structure of Retailers

Similar to Proposition 3, Proposition 4 shows that when one retailer $x$ offers a retail platform, only if the product substitutability $\gamma$ is relatively high and the store substitutability $\beta$ is low, the traditional channel is beneficial to retailer $y$. However, it is worth noting that the range of $(\beta, \gamma)$ for traditional channel decreases when the rival shifts from a traditional channel to a retail platform.

Both Proposition 3 and Proposition 4 explains the reason why the percentage of products among different categories sold by JD.com itself varies greatly. For products in electronics category, the product substitutability is relatively high because of the standard production and less differentiated usage, while the store substitutability is low since JD.com has a great reputation in selling electronics, so it is reasonable for JD.com to sell most of electronics products itself. But for products in Cosmetics category where the product substitutability is not relatively high or for products in Toys \& Games, Sports \& Outdoors, Shoes category where both the product substitutability and the store substitutability are sufficiently high, provides a retail platform for the third-party sellers is better choice for JD.com.

In terms of the conclusions above, it can be found that:

(i) if $(\gamma, \beta) \in L_{1}$, then $R_{i y}^{T P}>R_{i y}^{T T}, R_{i x}^{P P}>R_{i x}^{T P}, R_{i x}^{P P}>R_{i x}^{T T}$;

(ii) if $(\gamma, \beta) \in L_{2}$, then $R_{i y}^{T P}>R_{i y}^{T T}, R_{i x}^{P P}>R_{i x}^{T P}, R_{i x}^{P P}<R_{i x}^{T T}$;

(iii) if $(\gamma, \beta) \in L_{3}$, then $R_{i y}^{T P}<R_{i y}^{T T}, R_{i x}^{P P}>R_{i x}^{T P}, R_{i x}^{P P}<R_{i x}^{T T}$;

(iv) if $(\gamma, \beta) \in L_{4}$, then $R_{i y}^{T P}<R_{i y}^{T T}, R_{i x}^{P P}<R_{i x}^{T P}, R_{i x}^{P P}<R_{i x}^{T T}$. 
The following proposition establishes the equilibrium channel structure choice game of two retailers.

\section{Proposition 5}

(i) For given $(\gamma, \beta)$ in $L 1$, the retail platform PP is the subgame-perfect equilibrium for retailers.

(ii) For given $(\gamma, \beta)$ in L2, the retail platform PP is still the unique Nash equilibrium, but it is the classical prisoner's dilemma.

(iii) For given $(\gamma, \beta)$ in $L 3$, there are two equilibrium channels TT and PP. PP is the dominate equilibrium and TT is the classical prisoner's dilemma.

(iv) For given $(\gamma, \beta)$ in L4, the traditional channel TT is the subgame-perfect equilibrium for retailers.

Proposition5 indicates that when (1) the product substitutability $\gamma$ is sufficiently low, or(2) both the product substitutability $\gamma$ and the store substitutability $\beta$ are low, the retail platform is the dominant strategy for both retailers, and the unique equilibrium of channel game is PP. When both the product substitutability $\gamma$ and the store substitutability $\beta$ are relatively high, the PP is still the unique equilibrium, but it leads retailers into prisoner's dilemma. That is because the retailers can obtain more profits under a traditional channel than under a retail platform. When the product substitutability $\gamma$ is relatively high and the store substitutability $\beta$ is moderate, both TT and PP are the Nash equilibrium. However, TT is the optimal option for two retailers, and PP will lead the retailers into classical prisoner's dilemma. When the product substitutability $\gamma$ is relatively high and the store substitutability $\beta$ is relatively low, the TT is the unique Nash equilibrium and the best option for both retailers.

The above conclusions imply that the equilibrium channel structure of both retailers will gradually switch from retail platform to traditional retailing as the competitive intensity increases. If the competition intensity of product is relatively small, for example, TaoBao is almost a monopoly online retailer of China at the beginning of twenty-first century, and then platform-sell is the best option for it. As the degree of product substitutability increases, the traditional retailing is the only format for two manufacturers with relatively lower degree of store substitutability. That is, as the number of retail platforms increase, traditional resell will benefit to retailers. For example, in recent years, JingDong's products in the electronics category get a larger market share than TaBao.com, DangDang.com, and VIP.com.

\section{Competing Supply Chain Model with Product Service}

The previous section has focused on channel choice without considering product service. Here, we consider that the supply chain players who sell products to consumers will offer the products service, such as consultation, delivery, replacement etc. we investigate how the product competition and the product service affect the channel choice.

The demand function is $p_{i x}=a-\left(q_{i x}+\beta q_{i y}\right)-\gamma\left(q_{j x}+b q_{j y}\right)+k s_{i x}$, where $i=1,2 ; x=1,2 ; j=3-i ; y=3-x, k \geq 0$ represents the service-sensitivity of the products, $s \geq 0$ represents the product service level. Providing service level $s$ need invest cost $s^{2} / 2$. This assumption has been employed by prior literatures (e.g.,Lal 1990; Bhardwaj 2001). Section 6.1 presents the players' strategic behaviors in different channel configurations; section 6.2 identifies the equilibrium channel choice with product service offered. 


\subsection{The Strategic Behavior of Supply Chain Players}

When the product service is offered, two retailers give the channel structure to manufacturers firstly: traditional channel or retail platform. If the traditional channel is offered, the manufacturer sets wholesale price, and then retailer decides retail price and service level. If the retail platform is offered, the corresponding platform fee is given by retailer. If the manufacturers refuse it, then retailer and manufacturers only trade with traditional channel. If the manufacturers accept it, the platform agreement is achieved. The manufactures determine the retail price and service level. In the configuration TT, the retailer and manufacturer maximize their own profit respectively, as shown below:

$$
\begin{aligned}
& \pi_{M i}=w_{i x} q_{i x}+w_{i y} q_{i y} \quad i=1,2 ; j=3-i, \\
& \pi_{R x}=\left(p_{i x}(q)-w_{i x}\right) q_{i x}+\left(p_{j x}(q)-w_{j x}\right) q_{j x}-s_{i x}^{2} / 2-s_{j x}^{2} / 2 \quad x=1,2 ; y=3-x .
\end{aligned}
$$

In the configuration PP, the retailer and manufacturer's problems are as follows:

$$
\begin{aligned}
& \pi_{M i}=p_{i x}(q) q_{i x}+p_{i y}(q) q_{i y}-s_{i x}^{2} / 2-s_{i y}^{2} / 2, \quad i=1,2 ; j=3-i \\
& \pi_{R x}=2 F_{x} \quad x=1,2 ; y=3-x .
\end{aligned}
$$

In the configuration TP, the retailer and manufacturer's problems are as follows:

$$
\begin{aligned}
& \pi_{M i}=w_{i x} q_{i x}+p_{i y}(q) q_{i y}-F, \quad i=1,2 ; j=3-i, \\
& \pi_{R x}=\left(p_{i x}(q)-w_{i x}\right) q_{i x}+\left(p_{j x}(q)-w_{j x}\right) q_{j x}, \quad x=1,2 ; y=3-x .
\end{aligned}
$$

According to supply chain players' profit functions in different configurations, we can solve the Nash equilibrium and show it in Lemma1.

Lemma1 ( $i)$ with the channel structure TT, there exists a unique Nash equilibrium given by $\left\{\begin{array}{l}w_{i x}^{T T^{*}}=w_{i y}^{T T^{*}}=w_{j x}^{T T^{*}}=w_{j y}^{T T^{*}}=a\left(\left(2-k^{2}\right)^{2}-4 \gamma^{2}\right) / A_{1} \\ q_{i x}^{T T^{*}}=q_{i y}^{T T^{*}}=q_{j x}^{T T^{*}}=q_{j y}^{T T^{*}}=a\left(2-k^{2}\right) / A_{1} \\ s_{i x}^{T T^{*}}=s_{i y}^{T T^{*}}=s_{j x}^{T T^{*}}=s_{j y}^{T T^{*}}=a k\left(2-k^{2}\right) / A_{1}\end{array}\right.$

(ii) With the channel structure TP, there exists a symmetric unique Nash equilibrium given by

$$
\left\{\begin{array}{l}
w_{i x}^{T P^{*}}=w_{j x}^{T P^{*}}=a\left(b^{2}\left(k^{2} \gamma+2 k^{2}+2 \gamma^{2}-2 \gamma-4\right)+b\left(2 k^{2} \gamma-k^{4} \gamma+4 \gamma^{3}+4 \gamma^{2}\right)+\eta_{1}\right) / A_{2} \\
q_{i x}^{T P^{*}}=q_{j x}^{T P^{*}}=a k^{2}\left(2-k^{2}\right)\left(2-k^{2}-2 \beta+\gamma-\beta \gamma\right) / A_{2} \\
q_{i y}^{T P^{*}}=q_{j y}^{T P^{*}}=a\left(2 k^{4}+k^{2}(\beta \gamma+2 \beta-8-2 \gamma)+8-4 \beta+4 \gamma-4 \gamma^{2}-2 \beta \gamma+2 \beta \gamma^{2}\right) / A_{2} \\
s_{i x}^{T P^{*}}=s_{j x}^{T P^{*}}=a k^{3}\left(2-k^{2}\right) / A_{2} \\
s_{i y}^{T P^{*}}=s_{j y}^{T P^{*}}=a k\left(2 k^{4}-k^{2} \gamma-6 k^{2}-2 \gamma^{2}+2 \gamma+4\right) / A_{2}
\end{array}\right.
$$

(iii) With the channel structure PP, there exists a unique symmetric Nash equilibrium given by

$$
\left\{\begin{array}{l}
q_{i x}^{P P^{*}}=q_{i y}^{P P^{*}}=q_{j x}^{P P^{*}}=q_{j y}^{P P^{*}}=a /\left(2-k^{2}+2 \beta+\gamma+\beta \gamma\right) \\
s_{i x}^{P P^{*}}=s_{i y}^{P P^{*}}=s_{j x}^{P P^{*}}=s_{j y}^{P P^{*}}=a k /\left(2-k^{2}+2 \beta+\gamma+\beta \gamma\right)
\end{array}\right.
$$

Such that,

$$
\begin{aligned}
& A_{1}=2 \beta+4 \gamma+2 \beta \gamma-\beta k^{2}-2 k^{2} \gamma-8 k^{2}+2 k^{4}-4 \gamma^{2}-\beta k^{2} \gamma+8 \\
& A_{2}=k^{2}\left(\beta^{2} \gamma^{2}+4 \beta^{2} \gamma+4 \beta^{2}+2 \gamma^{2}-16 \gamma-24\right)+4 k^{4}(\gamma+3)-2 k^{6}-4 \gamma^{3}-4 \gamma^{2}+16 \gamma+16 \\
& \eta_{1}=\gamma^{2}\left(4 k^{2}-8\right)+\gamma\left(k^{4}-4 k^{2}+4\right)-4 \gamma^{3}+8-12 k^{2}+6 k^{4}-k^{6}
\end{aligned}
$$


Let the platform fees $F_{a}^{*}$ denote the threshold values of $\pi_{R y}^{T T^{*}}=\pi_{R y}^{T P^{*}}, F_{b}^{*}$ denote the threshold values of $\pi_{R x}^{P P^{*}}=\pi_{R x}^{T P^{*}}$ and $F_{c}^{*}$ denote the threshold values of $\pi_{R x}^{P P^{*}}=\pi_{R x}^{T T^{*}}$. Then, we can obtain:

$$
\left\{\begin{array}{l}
F_{a}^{*}=a^{2}\left(\beta^{3} \eta_{4}+\beta^{2} \eta_{5}+\beta \eta_{6}+\eta_{7}\right) /\left(2 A_{2}^{2}\right)-a^{2}\left(2-k^{2}\right)\left(\left(2-k^{2}\right)^{2}-4 \gamma^{2}\right) / A_{1}^{2} \\
F_{b}^{*}=a^{2}\left(2+2 \beta-k^{2}\right) / A_{3}^{2}-a^{2}\left(\beta^{3} \eta_{4}+\beta^{2} \eta_{5}+\beta \eta_{6}+\eta_{7}\right) /\left(2 A_{2}^{2}\right) \\
F_{c}^{*}=a^{2}\left(2+2 \beta-k^{2}\right) / A_{3}^{2}-a^{2}\left(2-k^{2}\right)\left(\left(2-k^{2}\right)^{2}-4 \gamma^{2}\right) / A_{1}^{2}
\end{array}\right.
$$

The following proposition compares the optimal quantity and service level in different configurations.

\section{Proposition 6}

(i) $q_{i y}^{T P^{*}}\left(q_{i x}^{P T^{*}}\right)>q_{i x}^{P P^{*}}>q_{i x}^{T T^{*}}>q_{i x}^{T P^{*}}\left(q_{i y}^{P T^{*}}\right)$.

(ii) $s_{i y}^{T P^{*}}\left(s_{i x}^{P T^{*}}\right)>s_{i x}^{P P^{*}}>s_{i x}^{T T^{*}}>s_{i x}^{T P^{*}}\left(s_{i y}^{P T^{*}}\right)$

Proposition 6 demonstrates if the manufacturers or retailers offer the product service to consumers, the comparing result of sale quantity for different configurations is the same as without offering product service. The retailer who chooses the retail platform in of model TP can have the highest sale quantity for all configurations. In addition, the manufactures' service level in the retail platform is always higher than in traditional channel. Therefore, manufacturers selling products on a platform is beneficial to develop products' brands, because they can offer expert service to consumers.

We give the equilibrium profits of manufacturers and retailer in different configurations in Table 3.

Table 3. The Equilibrium Prices and Profits with Product Service

\begin{tabular}{rl|l|l|l|}
\hline & TT & TP & PP \\
\hline$\pi_{M i}^{*}$ & $a^{2}\left(2-k^{2}\right)\left(\left(2-k^{2}\right)^{2}-4 \gamma^{2}\right) / A_{1}^{2}$ & $a^{2}\left(\beta^{3} \eta_{2}+\beta^{2} \eta_{3}+\beta \eta_{4}+\eta_{5}\right) /\left(2 A_{2}^{2}\right)-F$ & $a^{2}\left(2+2 \beta-k^{2}\right) / A_{3}^{2}-2 F$ \\
\hline$\pi_{M j}^{*}$ & $a^{2}\left(2-k^{2}\right)\left(\left(2-k^{2}\right)^{2}-4 \gamma^{2}\right) / A_{1}^{2}$ & $a^{2}\left(\beta^{3} \eta_{2}+\beta^{2} \eta_{3}+\beta \eta_{4}+\eta_{5}\right) /\left(2 A_{2}^{2}\right)-F$ & $a^{2}\left(2+2 \beta-k^{2}\right) / A_{3}^{2}-2 F$ \\
\hline$\pi_{R x}^{*}$ & $a^{2}\left(2-k^{2}\right)\left(2-k^{2}+2 \gamma\right) / A_{1}^{2}$ & $a^{2} k^{4}\left(2-k^{2}\right)^{2}\left(2-k^{2}+2 \gamma\right) / A_{2}^{2}$ & $2 F$ \\
\hline$\pi_{R y}^{*}$ & $a^{2}\left(2-k^{2}\right)\left(2-k^{2}+2 \gamma\right) / A_{1}^{2}$ & $2 F$ & $2 F$ \\
$A_{1}=$ & $2 \beta+4 \gamma+2 \beta \gamma-\beta k^{2}-2 k^{2} \gamma-8 k^{2}+2 k^{4}-4 \gamma^{2}-\beta k^{2} \gamma+8$ \\
$A_{2}=$ & $k^{2}\left(\beta^{2} \gamma^{2}+4 \beta^{2} \gamma+4 \beta^{2}+2 \gamma^{2}-16 \gamma-24\right)+4 k^{4}(\gamma+3)-2 k^{6}-4 \gamma^{3}-4 \gamma^{2}+16 \gamma+16$ \\
$A_{3}=$ & $2-k^{2}+2 \beta+\gamma+\beta \gamma$ \\
$\eta_{2}=$ & $4 k^{4}(\gamma+2)^{2}+4 k^{2}\left(\gamma^{4}+6 \gamma^{3}+4 \gamma^{2}-16 \gamma-16\right)+8 \gamma^{5}+8 \gamma^{4}-48 \gamma^{3}-48 \gamma^{2}+64 \gamma+64$ \\
$\eta_{3}=$ & $3 k^{6}\left(4-\gamma^{2}\right)+k^{4}\left(8 \gamma^{3}+30 \gamma^{2}-24 \gamma-72\right)+4 k^{2}\left(36-12 \gamma^{3}-21 \gamma^{2}+24 \gamma\right)-8\left(3 \gamma^{5}-4 \gamma^{4}-8 \gamma^{3}-9 \gamma^{2}+12 \gamma+12\right)$ \\
$\eta_{4}=$ & $-8 k^{8}(\gamma+1)+8 k^{6}\left(\gamma^{2}+8 \gamma+8\right)+8 k^{4}\left(2 \gamma^{3}-\gamma^{2}-24 \gamma-24\right)-8 k^{2}\left(\gamma^{4}+10 \gamma^{3}+8 \gamma^{2}-32 \gamma-32\right)+16\left(\gamma^{5}+\gamma^{4}+6 \gamma^{3}\right.$ \\
& $\left.+6 \gamma^{2}-8 \gamma-8\right)$ \\
$\eta_{5}=$ & $-6 k^{10}+12 k^{8}(\gamma+5)+6 k^{6}\left(3 \gamma^{2}-16 \gamma-40\right)+4 k^{4}\left(120-8 \gamma^{3}-27 \gamma^{2}+144 \gamma\right)+k^{2}\left(-8 \gamma^{4}+128 \gamma^{3}+216 \gamma^{2}-384 \gamma\right.$ \\
& $-480)+16 \gamma^{4}-128 \gamma^{3}-144 \gamma^{2}+192 \gamma+192$
\end{tabular}

Proposition 7 Given that the product service is offered, if two manufacturers are independent, i.e., $\gamma=0$, then,

(i) If $0<k<k_{1}(\beta)<1.414$, then $R_{i y}^{T P}>R_{i y}^{T T}, R_{i x}^{P P}>R_{i x}^{T P}, R_{i x}^{P P}>R_{i x}^{T T}$..

(ii) If $1<k_{1}(\beta)<1.414$, then $R_{i y}^{T P}>R_{i y}^{T T}, R_{i x}^{P P}>R_{i x}^{T P}, R_{i x}^{P P}>R_{i x}^{T T}$.

Such that $k_{1}=\sqrt{2-\beta}$. 
Proposition7 shows when the product service is offered and the two products are independent, the retailer's channel choice relies heavily on the service-sensitivity. If the service-sensitivity $k$ is low, i.e., $0<k<k_{1}(\beta)<1.414$, the retail platform is the best option for both retailers, and the unique equilibrium of channel game is PP. When the service-sensitivity $k$ is relatively high, the PP is still a unique equilibrium, but it lead to retailers in prisoner's dilemma. That is because the retailers can obtain more profits under a traditional channel than under a retail platform.

\subsubsection{Imperfect Substitute}

Next proposition we discuss the case that two products offered by two different manufacturers are imperfect substitute, i.e., $\gamma=1 / 2$.

Proposition 8 when the product service is offered, if two manufacturers are imperfect substitute, i.e., $\gamma=1 / 2$, then,

(i) If $0<k<k_{2}(\beta)<1.121$, then $R_{i y}^{T P}>R_{i y}^{T T}, R_{i x}^{P P}>R_{i x}^{T P}, R_{i x}^{P P}>R_{i x}^{T T}$.

(ii) If $0.833<k_{2}(\beta)<k<1.121$, then $R_{i y}^{T P}>R_{i y}^{T T}, R_{i x}^{P P}>R_{i x}^{T P}, R_{i x}^{P P}<R_{i x}^{T T}$.

Such that $k_{2}=\left(9-2 \sqrt{\left(4 \beta^{2}-4 \beta+17\right)^{1 / 2}-2 \beta}\right) / 4$.

Proposition9 suggests that when the product service is offered and the two products is imperfect substitute, if the service-sensitivity $k$ is under a certain value $k_{2}$, the retailers' profit under the retail platform is more than under the traditional platform. When the service-sensitivity $k$ is relatively high, the retailers still prefer the retail platform, but it lead to retailers in prisoner's dilemma.

\subsubsection{Perfect Substitute}

Next proposition we discuss the case that two products offered by two different manufacturers are perfect substitute, i.e., $\gamma=1$.

Proposition 9 when the product service is offered, if two manufacturers are perfect substitute, i.e., $\gamma=1$, then,

(i) If $0<k<k_{3}(\beta)<0.665$, then $R_{i y}^{T P}>R_{i y}^{T T}, R_{i x}^{P P}>R_{i x}^{T P}, R_{i x}^{P P}>R_{i x}^{T T}$.

(ii) If $0<k_{3}(\beta)<k<0.665$, then $R_{i y}^{T P}>R_{i y}^{T T}, R_{i x}^{P P}>R_{i x}^{T P}, R_{i x}^{P P}<R_{i x}^{T T}$.

Such that $k_{3}=\sqrt{2\left(5-\left(\beta^{2}-2 \beta+17\right)^{1 / 2}-\beta\right.} / 2$.

Proposition9 indicates that the case that two products are perfect substitute is similar as the case that two products are independent or imperfect substitute. PP is the unique Nash equilibrium for retailers. When the service-sensitivity $k$ is low, the retail platform is the dominant stategy, When the service-sensitivity $k$ is high, the PP is the prisoner's dilemma.

Proposition10 the range of service-sensitivity $\left(0, k_{1}\right),\left(0, k_{2}\right)$ and $\left(0, k_{3}\right)$ in which the retail platform is the dominant equilibrium is decreasing in store substitutability $\beta$. In contrast, the range of service-sensitivity $\left(k_{1}, 1\right),\left(k_{2}, 1\right)$ and $\left(k_{3}, 1\right)$ in which the retail platform is the prisoner's dilemma is increasing in store substitutability $\beta$.

Proposition10 implies that with the product service offered, the retail platform is a unique equilibrium choice for retailers. We illustrate it in Figure 3. When the product substitutability is under a certain value, i.e., $k_{1}$, the platform is the dominant strategy. However, when the product substitutability exceeds the certain value $k_{1}$, the retail 
platform will make the retailers in classical prisoner's dilemma. The value $k_{1}$ is decreasing in store substitutability $\beta$. In the other word, as store substitutability $\beta$ increases, the retail platform is beneficial to the retailers (see figure3).

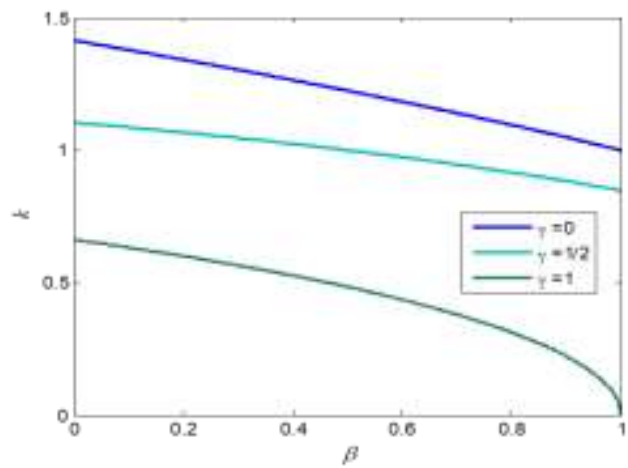

\section{Figure 3. the Effect of Product Substitutability On Channel Chain Retailers}

These observations demonstrate that the range for retail platform being a dominant strategy decreases as the degree of product competition increases. It implies that when the product service is offered, the channel choice of retailer is not only dependent on servicesensitivity, but also effected by product and store substitutability. The former plays a decisive role, and the latter is the catalyst.

\section{Conclusion}

The retail platform becomes an important distribution format observed worldwide in the retailing industry. In the paper, we start by characterizing how the strategic behavior of supply chain members changes under different distribution channels. According to different channel choice, we build three types of models: (i) the traditional channel competition model, in which both manufacturers sell products to retailers; (ii) the retail platform competition model, in which both manufactures sell products by a platform; (iii) the mixed channel competition model, in which two retailers choose different channels to distribute products. Comparing the models, we discuss the equilibrium channel structure and the dominant channel strategy for retailers. We find that without product service, the equilibrium channel choice of two retailers depends on both the product substitutability and store substitutability, and the product substitutability is the key factor to influence equilibrium outcome. With product service, the retailers' choice is affected by the servicesensitivity, product and store substitutability, but the service-sensitivity is the key factor. What's more, we examine the conditions of the retail platform as equilibrium structure. Without product service, when the product substitutability is sufficiently low, or both the product substitutability and the store substitutability are low, the retail platform is the subgame-perfect equilibrium. With product service, when service-sensitivity is low, the retail platform is a dominant strategy for retailers. When service-sensitivity is high, the retail platform will push the retailers into the prisoner's dilemma.

Our paper has several limitations that further research can address. We use a certainty and linear demand function in our paper. Although this demand function has been extensively used in the literature, it would lead to ignore some issues resulting from demand uncertainty. In addition, we focus on two symmetric retailers and manufacturers, but asymmetrically competing supply chain members might be more interesting. Another possible extension could consider the impact of consumer's preference on distribution channel. Despite these assumptions, we believe that we have addressed an important issue in the area of distribution channel under competing supply chain. 


\section{Acknowledgments}

The authors gratefully acknowledge the support grants from the National Natural Science Foundation of China (NSFC), (nos. 71402101, 71761020), the Ministry of education of the humanities and Social Sciences of China (nos. 16YJC630057)

\section{References}

[1] X. Z. Ai, J. Chen, J. H. Ma and H. X. Zhao, "Competition among supply chains: Implications of full returns policy", International Journal of Production Economics., vol. 129, no. 1, (2012), pp. 257-265.

[2] B.J. Jiang, K. Jerath and K. Srinivasan, "Firm Strategies in the Mid-Tail of Platform-Based Retailing", Marketing Science., vol. 30, no. 5, (2011), pp. 757-775.

[3] F. Bernstein and F. deVe'ricourt, "Competition for procurement contracts with service guarantees", Operations Research., vol. 56, no. 5, (2008), pp. 562-575.

[4] E. Brynjolfsson and M. Smith, "Frictionless commerce? A comparison of internet and conventional retailers", Management Science., vol. 46, no. 4, (2000), pp. 563-585.

[5] G. S. Cai, Y. Dai and S. X. Zhou, "Exclusive Channels and Revenue Sharing in a Complementary Goods Market”, Marketing Science., vol. 31, no. 1, (2012), pp. 172-187.

[6] K. Cattani, W. Gilland and H. S. Heese, "Boiling frogs: pricing strategies for a manufacturer adding a direct channel that competes with the traditional channel", Production and Operations Management., vol. 15, no. 1, (2006), pp. 40-56.

[7] A. T. Coughlan, "Competition and cooperation in marketing channel choice: theory and application", Marketing Science, vol. 4, no. 1, (1985), pp. 110-129.

[8] A. Dukes, E. Gal-Or and K. Srinivasan, "Channel bargaining with retailer asymmetry", Journal of Marketing Research., vol. 43, no. 1, (2006), pp. 84-97.

[9] N. Economides and S. C. Salop, "Competition and integration among complements, and network market structure", Journal of Industrial Economics., vol. 40, no. 1, (1992), pp. 105-123.

[10] F. Zettelmeyer, "Expanding to the Internet: Pricing and Communications Strategies When Firms Compete on Multiple Channels", Journal of Marketing Research, vol. 37, no. 3, (2000), pp. 292-308.

[11] A.Y. Ha and S. Tong, "Contracting and information sharing under supply chain competition", Management Science., vol. 54, no. 4, (2008), pp. 701-715.

[12] H. Horn and A. Wolinsky, "Bilateral monopolies and incentives for merger", The RAND Journal of Economics., vol. 19, no. 3, (1988), pp. 408-419.

[13] C. A. Ingene and M. E. Parry, "Mathematical Models of Distribution Channels", Springer, New York., vol. 10 , no. 23, (2004), pp. 14-16.

[14] R. Lal and M. Sarvary, "When and how is the Internet likely to decrease price competition?", Marketing Science, vol. 18, no.4, (1999), pp. 485-503.

[15] T.W. McGuire and R. Staelin, "An industry equilibrium analysis of downstream vertical integration", Marketing Science, vol. 2, no.2, (1983), pp. 161-191.

[16] K.S. Moorthy, "Strategic decentralization in channels", Marketing Science., vol. 7, no.3, (1988), pp. 335-355.

[17] S. Moorthy, "A general theory of pass-through in channels with category management and retail competition", Marketing Science, vol. 24, no.5, (1983), pp. 110-122.

[18] D. P. O'Brien and G. Shaffer, "Vertical control with bilateral contracts", The RAND Journal of Economics, vol. 23, no.3, (1992), pp. 299-308.

[19] D. P.O. 'Brien and G. Shaffer, "Bargaining, bundling, and clout: The portfolio effects of horizontal mergers", The RAND Journal of Economics, vol. 36, no.3, (1983), pp. 573-595.

[20] E. Ofek, Z. Katona and M. Sarvary, "Bricks and Clicks: The Impact of Product Returns on the Strategies of Multichannel Retailers", Management Science., vol. 30, no.1, (2011), pp. 42-60.

[21] S Balasubramanian, "Mail versus Mall: A Strategic Analysis of Competition between Direct Marketers and Conventional Retailers", Marketing Science., vol. 17, no.3, (1988), pp.181-195.

[22] M. Trivedi, "Distribution channels: an extension of exclusive retailership", Management Science., vol. 44, no.7, (1998), pp. 896-909.

[23] Y. Wei, C. Kevin, D. Chhajed and J D. Hess, "Direct Marketing, Indirect Profits: A Strategic Analysis of Dual-Channel Supply Chain Design", Management Science., vol. 49, no.1, (2003), pp. 1-20.

[24] W. S. Yoo and E. Lee, "Internet Channel Entry: A Strategic Analysis of Mixed Selling formats", Management Science, vol. 30, no.1, (2011), pp. 29-41.

[25] S. Ziss, "Vertical separation and horizontal mergers", Journal of Industrial Economics, vol. 43, no.1, (1995), pp. 63-75. 